\title{
Género y relaciones capitalistas de producción: una reflexión desde la perspectiva marxista
}

\author{
Arelys Esquenazi Borrego ${ }^{1}$ \\ https://orcid.org/0000-0002-9366-8688 \\ ${ }^{1}$ Universidade Federal do Espírito Santo, Programa de Pós-Graduação em Política Social, Vitória, ES, Brasil
}

Género y relaciones capitalistas de producción: una reflexión desde la perspectiva marxista

Resumen: Este artículo tiene como objetivo analizar la vinculación existente entre las relaciones de género y las relaciones capitalistas de producción. Este análisis tiene como idea central defender que la producción-reproducción social en el Capitalismo genera necesariamente, como condición y resultado, relaciones sociales de explotación de clase y de opresión de género. En este sentido, se destaca la necesidad de entender la categoría género, a nivel esencial, como una relación social de producción. De igual forma, se analizan diferentes concepciones de género, a partir de destacar algunos mitos/fetiches asociados a esta temática. Posteriormente, se analiza desde un plano más esencial las diferentes interrelaciones que se establecen entre relaciones capitalistas de producción y relaciones de género; así como, su expresión en la etapa actual de la globalización neoliberal. La crítica de la Economía Política constituye el fundamento metodológico de los análisis desarrollados a lo largo del artículo.

Palabras clave: Género. Fetichismo. Explotación/Opresión. Relaciones capitalistas de producción. Globalización neoliberal.

Gênero e relações capitalistas de produção: uma reflexão a partir da perspectiva marxista

Resumo: Este artigo tem como objetivo analisar a relação entre as relações de gênero e as relações capitalistas de produção. Esta análise tem como idéia central defender que a produção-reprodução social no capitalismo gera, necessariamente, como condição e resultado, relações sociais de exploração de classe e de opressão de gênero. Nesse sentido, destaca-se a necessidade de compreender a categoria de gênero, em nível essencial, como relação de produção social. Da mesma forma, diferentes concepções de gênero são analisadas, a partir do destaque de alguns mitos/fetiches associados a esse tema. Posteriormente, as diferentes inter-relações estabelecidas entre as relações capitalistas de produção e as relações de gênero são analisadas a partir de um ponto de vista mais essencial; bem como sua expressão no atual estágio da globalização neoliberal. A crítica da Economia Política constitui o fundamento metodológico das análises desenvolvidas ao longo do artigo.

Palavras-chave: Gênero. Fetichismo. Exploração/Opressão. Relações capitalistas de produção. Globalização neoliberal.

\section{Gender and capitalist relations of production: a reflection from a Marxist perspective}

Abstract: This article aims to analyze the link between gender relations and capitalist production. The central idea is to explain that social production-reproduction in Capitalism necessarily generates, as a condition and result, social relations of class exploitation and gender oppression. In this sense, the need to understand gender, in its essence as a relationship of social production is highlighted. Similarly, different conceptions of gender are analyzed, highlighting some associated myths/fetishes. Subsequently, the different interrelationships established between capitalist relations of production and gender relations are analyzed from a more essential point of view; as well as its expression in the current stage of neoliberal globalization. The critique of Political Economy constitutes the methodological foundation of the analyzes developed throughout the article.

Keywords: Gender. Fetishism. Exploitation/Oppression. Capitalist production relations. Neoliberal globalization.

Recibido en 12.10.2017. Aprobado en 08.02.2018. Revisado en 21.06.2018.

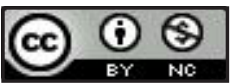

(C) El(Los) Autor(es). 2018 Acceso Abierto Esta obra está licenciada bajo los términos de la Licencia Creative Commons Atribución-NoComercial 4.0 Internacional (https://creativecommons.org/licenses/by-nc/4.0/deed.es), que permite copiar, distribuir y reproducir en cualquier medio, así como también adaptar, transformar y crear a partir de este material, desde que para fines no comerciales, y que usted fornezca el crédito debido a los autores y a la fuente, insiera un enlace para la Licencia Creative Commons e indique si fueron hechas alteraciones. 
(...) el cambio de una época histórica puede determinarse siempre por la actitud de progreso de la mujer ante la libertad, ya que es aquí, en la relación entre la mujer y el hombre, entre el débil y el fuerte, donde con mayor evidencia se acusa la victoria de la naturaleza humana sobre la brutalidad. El grado de la emancipación femenina constituye la pauta natural de la emancipación general. (Fourier, 1841 apud Marx \& Engels, 1844, p. 261).

\section{Introducción}

El presente artículo no pretende realizar un paralelismo entre teorías marxistas y teorías feministas o de género; es decir, ver como las segundas incorporan la visión del marxismo, o como el marxismo ha abordado directa o indirectamente la cuestión de género. Esta línea de investigación no se suscribe en su totalidad, pues supone segmentar o dividir el marxismo por temas, lo cual implica desconocer o subvalorar el objeto y el método de estudio del marxismo como paradigma de ciencia. Es innecesario hablar de una teoría marxista de género como si fuese una nueva corriente; mejor sería decir que el marxismo, a partir de su propuesta de análisis y transformación de la sociedad con base en un enfoque de totalidad, permite analizar bajo su prisma una gran diversidad de temas, entre los que se pudiera mencionar la temática de género.

De esta forma, la elección del tópico de género, y su reflexión crítica a partir de algunas categorías centrales del marxismo, tiene como intención contribuir al diálogo entre ambas corrientes; y especialmente reivindicar al paradigma marxista como ciencia e ideología de la interpretación, crítica y transformación revolucionaria de la realidad.

En este sentido, el propósito de estas líneas es, en un primer momento, el de realizar una mirada reflexiva desde el marxismo a las concepciones de género imperantes en la sociedad capitalista contemporánea, a partir de la utilización de categorías centrales para dicha corriente como: ideología, relaciones sociales de producción, relaciones de explotación, clases sociales, fetichismo. Por otra parte, dicha reflexión busca aproximarse a las conexiones que se establecen entre las relaciones de género y las relaciones capitalistas de producción; y a su vez, se busca destacar las interrelaciones existentes entre las desigualdades de género y la contradicción capital-trabajo. Este análisis tiene como objetivo central defender la idea de que la producción-reproducción social en el Capitalismo, genera necesariamente como condición y resultado relaciones sociales de explotación de clase y de opresión de género, estrechamente articuladas entre sí; por cual la categoría debe ser también entendida a un nivel más esencial como expresión de relaciones sociales de producción.

Para ello el presente artículo ha sido estructurado en tres acápites. En un primero momento, se analizan críticamente las concepciones imperantes de género, a través de analizar las mistificaciones/fetiches que las caracterizan, oponiendo su apariencia y esencia. Posteriormente, se destacan los nexos entre las relaciones de género y de las relaciones capitalistas de producción. Por último, se abordan brevemente algunas de las principales características de la etapa actual de desarrollo del sistema capitalista; así como, las implicaciones de la llamada globalización neoliberal en la internacionalización de las desigualdades y opresiones de género.

Lo anteriormente comentado supondrá, esencialmente, asumir el método dialéctico materialista e histórico como fundamento epistemológico. A través de dicha orientación metodológica, se dialoga con la perspectiva de género a través del ejercicio/aplicación de la crítica de la Economía Política a esta temática.

\section{Concepciones de género: las contradicciones entre esencia y apariencia}

La primera idea a resaltar es que la construcción teórica alrededor del concepto de género es un proceso no concluido, un campo en desarrollo, que condensa interpretaciones o visiones muy heterogéneas. Por lo que no existe un único concepto de género, aunque si algunos relativos consensos al respecto.

El género tiene su base material en un fenómeno natural, de nacimiento que es el sexo. Es por ello, que el sexo condiciona pero no determina al género, pues este último trasciende la simple distinción de los individuos en cuanto a su condición biológica (femenino-masculino); es por tanto una categoría más abarcadora. A su vez, esta relación tiene que ser entendida en su doble condicionamiento; debido a que el sexo es también algo socialmente determinado. Asumir la dialéctica que se establece entre ambas categorías, implica tomar como punto de partida un concepto más complejo, que es el sistema sexo-género.

Una forma de simplificación, o fetichización común sobre el concepto de género, es precisamente homologar de forma errónea ambas categorías, sexo y género; así como, ver la relación que entre ellas se establece de manera unidireccional y no dialéctica. Dicha simplificación implica que, muchas veces, estudios que pretenden 
problematizar sobre cuestiones de género en la actualidad, se queden en la realidad sensitiva o aparencial de simplemente analizar estadísticas diferenciadas según sexo.

Asociada a esta visión estrecha, se encuentra también relacionada otra forma simplista de asumir el género, que es reducir o igualar este concepto a las mujeres; presentarlo solamente como una cuestión de mujeres. Esta asociación pudiera tener su origen en que las mujeres, y específicamente el movimiento feminista, fueron las primeras en utilizar dicho término (género), para hacer referencia a la situación de subordinación y discriminación que padecían. Este parece ser un error muy trivial, pero conlleva a implicaciones conceptuales y prácticas muy peligrosas, como por ejemplo el que las políticas públicas con perspectiva género (eslogan muy común en estos días), en muchos casos, solo estén orientadas hacia las féminas.

Una visión crítica sobre este aspecto implicaría asumir al género como una categoría relacional - incluso no solamente asociada a la forma en que se concretan relaciones entre hombres y mujeres - y no como un concepto unitario y estático. Significaría también, tener una visión multidimensional y multicausal del fenómeno, que estaría definiéndose y expresándose a través de diversos aspectos, en estrecha relación entre sí, tales como elementos de tipo: culturales, históricos, biológicos, sociales, institucionales, políticos, psicológicos, jurídicos, económicos, entre otros.

Otro aspecto simplificador es asociar el género solamente a la discriminación o desigualdad femenina, reducirlo a un grupo vulnerable/desventaja específico. Lo anteriormente defendido, no implica desconocer las relaciones de poder entre los géneros y las relaciones de clase sobre las que se desarrollan, y cómo estas se encuentran marcadas por un orden imperante que produce y reproduce constantemente desigualdad social, económica, de derechos, de roles/responsabilidades, etc.; que se concretan en estereotipos y preconceptos tan alienantes como el que se está criticando en este mismo momento. En este sentido, es importante aclarar que la categoría género, per se, no implica necesariamente diferencias en detrimento de las mujeres; estas son resultado, expresión de la forma en que se han asumido y concretado dichas diferencias en la sociedad; es decir, su expresión histórico-concreta.

Es reduccionista ver por tanto al género, automáticamente como expresión de inferioridad, de discriminación y de desigualdad. Por eso, se insiste en que más que una definición acabada de género, es mejor hablar en términos de categoría relacional en constante evolución; que es expresión de cómo se asumen y se construyen socialmente las relación de los géneros y entre los géneros; tanto desde las relaciones de propiedad, como desde las relaciones de clases, o las relaciones de poder; así como, a través de qué formas se concretan un conjunto amplio de relaciones asociadas a la superestructura social (relaciones familiares, religiosas, jurídicas, entre otras).

De esta forma, la expresión concreta (específica) de un sistema de género caracterizado por dichos niveles de desigualdad y opresión en detrimento de la mujer, es lo que se conoce como patriarcado. El mismo delimita los espacios públicos (masculinos) con valor económico, social y de prestigio; y los espacios privados (femeninos) en el hogar, de servicio hacia la familia, invisibilizados y sin valor económico socialmente reconocido. Lo alienante es que muchas veces esto se nos presenta como lo común, como el deber ser, y obviamos que detrás de estas formas de organización patriarcal (producción-reproducción ${ }^{1}$ de la vida social) se fundamentan relaciones sociales de explotación y opresión.

Sin embargo, tan enajenante como lo que se acaba de apuntar, es creer que la lucha por la igualdad de género, es una lucha sólo de las mujeres. Pensar que acabar con el patriarcado posibilitará lograr la emancipación plena (política-económica-social) de la mujer, es tan ilusorio con acreditar que este proceso es de hecho posible a través de reformas dentro del sistema capitalista. Por ello, se insiste en que no se puede solamente combatir la desigualdad, los preconceptos y la opresión de género/sexo, sino se combaten al mismo tiempo las causas esenciales que las generan. No se trata de luchar en última instancia contra la división sexual del trabajo, el patriarcado, el machismo o la discriminación; porque se estarían combatiendo los efectos y no las determinaciones o causas esenciales que asumen formas de opresión en el capitalismo. 
Por último, otro aspecto fetichista es analizar el género desde una perspectiva dicotómica, binaria. Esto se concreta en una construcción social, o valoración de género que establece, y constantemente reproduce, una demarcación de roles donde las niñas y las mujeres se asociación con: la feminidad, lo femenino, la emoción, la sensibilidad, la debilidad y lo inferior; en contraposición a una visión de los niños y los hombres que se asocia comúnmente con: la masculinidad, lo masculino, la razón, la fuerza, lo superior.

El fetichismo es tal, que se asumen estas formas dicotómicas como lo natural. Se olvida que el concepto de género alude al conjunto de características, comportamientos, roles, funciones y valoraciones impuestas (he aquí el énfasis más importante) dicotómicamente a cada sexo a través de procesos de socialización, mantenidos y reforzados por: la tradición, el sentido común, la política, la religión, la ideología dominante (de la clase dominante), la forma en que se cuenta la historia de la humanidad, por las relaciones de poder y de clase, y por diferentes instituciones patriarcales (sistema educativo, derecho, iglesia, etc.).

En otras palabras se olvida que el género es resultado de relaciones sociales que se encuentran temporal y espacialmente determinadas. Debido a esta interpretación del género como resultado de relaciones sociales, es que se ha desarrollado otro concepto relativamente más reciente que es el sistema de género social. El cual se entiende como una especie de orden u estado de las cosas, una forma de legitimación de procesos, de mecanismos que regulan y organizan la sociedad de forma diferenciada para hombres y mujeres.

Por último, resaltar que el análisis de género que se desarrolla en este artículo toma como base una integración dialéctica entre los fenómenos de opresión y explotación que se producen en el sistema capitalista, pero sobre la base de considerar a ambos como categorías diferentes. La explotación está siempre creando y alimentando nuevas formas de opresión (entre ellas las de género), es por ello que la finalidad no puede ser solo eliminar las formas de opresión, sino esencialmente las causas de la explotación.

La opresión es más visible y más fácil de reconocer que la explotación que, en el capitalismo, es oculta y disfrazada por el régimen burgués. La explotación, o su condición de mano de obra explotada, está tan naturalizada en la sociedad que no se percibe en cuanto tal. Al contrario, la condición de oprimido, es más evidente, como en el caso del machismo, racismo, homofobia, xenofobia. Sin embargo, la raíz primera y última de la opresión, de la existencia de sectores oprimidos, debe ser buscada en la explotación, o sea, en la división de la sociedad en clases, porque la propiedad privada es la base material que sustenta la opresión, y se ella fuera suprimida, la tendencia será a la supresión de las bases materiales que alimentaban la opresión. Lo contrario no es verdadero. Se conseguimos aliviar la opresión, combatir los preconceptos, podremos, como máximo, imponer una sociedad más fraterna, lo que es muy importante, pero no garantiza de forma alguna la supresión definitiva de las diversas formas de opresión. (Toledo, 2017, p. 167).

No se puede solo combatir la desigualdad y la opresión de género, sino se combaten al mismo tiempo las causas esenciales que la generan. Lo anterior no quiere decir que no sea importante o válido la lucha contra cualquier forma de discriminación o desigualdad de género, contra el machismo. Son importantes - en tanto permiten visibilizar la situación de la mujer, generar conciencia al respecto e inclusive permiten realizar alcanzar conquistas puntuales importantes en ciertos períodos - pero esto no puede hacer perder de vista que la finalidad de la trasformación tiene que ser la ruptura y superación de la lógica del capital. La lucha de clase debe guiar el camino para la verdadera emancipación de la mujer.

Un lector perspicaz debería cuestionarse de qué sirve este ejercicio de dilucidar la esencia y la apariencia acerca de las concepciones de género imperantes. En respuesta a esto, se podría considerar en que el investigador sagaz no demerita ninguno de los planos de manifestación del fenómeno. La apariencia por fetichizada que se muestre, constituye una unidad de lo real, por ello no debe ser tampoco desconsiderada. Ambos planos de análisis (esencia-apariencia, concreto-abstracto) aportan niveles diferenciados pero imprescindibles de conocimiento, ambos son necesarios para comprender lo complejo y fetichizado que se muestra el fenómeno, y por ende lo difícil, primero, de crear consciencia al respecto; y segundo, lo complicado de que sea transformado en percepción y en acción por parte de la sociedad.

Finalmente, desde el punto de vista de la autora, el género desde un plano de análisis más concreto o aparencial debe ser entendido como una categoría que tiene su base material en un fenómeno de nacimiento, que es el sexo; pero que a su vez se encuentra condicionado y se expresa de manera cultural, política, económica, psicológica, religiosa, jurídica, histórica, etc. Por su parte, desde un plano más esencial puede ser interpretado como el conjunto de características y comportamientos que se atribuyen y se consideran permitidos y valorados para cada sexo; los cuales varían de acuerdo a contextos espaciales y temporales, por lo tanto, es necesario entenderlo en su evolución histórica, o como una categoría dinámica, en constante redefinición, y fundamentalmente relacional. Asumir el género como constructo social y relacional, implica no solo entender su 
condicionamiento a través de relaciones sociales - donde se constituyen reglas, normas y prácticas, por medio de las cuales se asignan recursos, tareas, responsabilidades y roles diferenciados - las cuales a su vez determinan las interrelaciones de los géneros y entre los géneros; sino también el condicionamiento a través de relaciones o formas de producción y reproducción imperantes en la sociedad.

Desde esta perspectiva más esencial, el género debe ser comprendido esencialmente como una relación social de producción, como un fenómeno que tiene su expresión tanto en la base económica, como en la superestructura de una sociedad, por lo cual debe ser analizado de forma transversal a todas estas esferas de actuación de la vida social. Esta idea se desarrolla con más profundidad a continuación.

\title{
Las relaciones de género y su conexión con la esencia de las relaciones capitalistas de producción
}

La contradicción capital-trabajo constituye el núcleo duro de la lógica del capital, a partir de la cual se derivan el resto de las contradicciones en el sistema capitalista. En este sentido, en la medida en que esta contradicción es gestionada a través de diversos modos, se convierte en una palanca, y freno, para el propio desarrollo de este modo de producción. Las formas más esenciales de concreción de dicha contradicción están asociadas a la relación que se establece entre reproducción-producción-propiedad-apropiación en el sistema capitalista. Las formas de manifestaciones aparenciales de la misma son diversas.

Por su parte, la temática de género se entrelaza a ambos niveles de análisis. De lo que trata en este punto es precisamente de reflexionar acerca de si las desigualdades de género en el mundo del trabajo pueden ser consideradas como parte de estas formas de manifestación fenoménicas de la contradicción capital-trabajo.

El presupuesto de partida es el de asumir las relaciones de género como relaciones de producción; lo cual implica en primera instancia, invalidar el fetiche de las relaciones de género solamente como relaciones entre hombres y mujeres, y comenzar a analizarlas como relaciones sociales, y más específicamente como relaciones sociales de producción. Según Haug (2006, p. 335):

\begin{abstract}
Pensar en relaciones de género como relaciones de producción puede parecer presuntuoso ya que acostumbramos a pensar en estas como parte de la organización de la producción de los medios de vida. Así es que entendemos las relaciones capitalistas de producción como una organización de producción provechosa y rentable para el mercado. Conceptos centrales para el análisis de estas relaciones, tales como el carácter dual de la labor, el trabajo alienado o asalariado y las fuerzas productivas, todos provienen de la economía y la política. Esta aproximación supone que todas estas determinaciones son género-neutro y, por tanto, que todas las prácticas que son género-específicas no están relacionadas con las relaciones de producción y son, como mucho, afectadas por ellas periféricamente.
\end{abstract}

En un segundo momento, este enfoque también implica reconocer la utilización de relaciones de género en el nivel agregado del conjunto de las relaciones de producción, es decir, en qué medida los sexos sirven para (re)producir el conjunto de las relaciones sociales de producción. Un punto de partida interesante es el análisis de cuáles son las implicaciones que tiene la separación de la esfera de la reproducción social, de la producción en el Capitalismo sobre las cuestiones de género.

La noción de que bajo el capitalismo, el modo de producción determina el modo de reproducción y, consecuentemente, relaciones desiguales observables entre hombres y mujeres, no es una forma de "economicismo" o un "reduccionismo de clase", sino el reconocimiento de la compleja red de efectos de nivel macro que actúa sobre las relaciones hombre-mujer, de un modo de producción impulsado por la acumulación de capital, en lugar de por el objetivo de satisfacer las necesidades de la gente. Sostener lo contrario, postulando la "mutua interacción" entre la organización de la producción y la organización de la reproducción, o dando primacía causal a esta última, es pasar por alto la importancia teórica de la abrumadora evidencia que documenta la subordinación capitalista de la reproducción a la producción. (Gimenez, 2005, p. 20).

En el libro primero de El Capital: Crítica de la Economía Política, Marx apunta que la conservación y reproducción de la clase trabajadora constituye una condición necesaria para la reproducción del capital. De conjunto a Engels en el escrito de 1844 de La Sagrada Familia, también va reflexionar sobre la importancia de comprender las relaciones entre modo de producción y modo de reproducción desde una perspectiva histórica. De esta forma, la reproducción adquiere matices más allá de lo puramente biológico. 
En el seno de toda formación social coexisten una producción social de bienes y una producción social de seres humanos. Producción y reproducción son indisociables. Para que el proceso de reproducción social capitalista funcione (lo que incluye la provisión de fuerza de trabajo), las relaciones de producción y de reproducción - en tanto componentes cruciales del proceso de reproducción social - deben evitar contradicciones entre sí. (Yañez, 2004, p. 64).

En este sentido, también se defiende la visión de que "[...] el Capitalismo se ha edificado sobre la división sexual del trabajo, que implica ganancias porque las mujeres contribuyen en forma gratuita o a muy bajo costo a la reproducción de la fuerza de trabajo para extraer plusvalía." (Anzorena, 2008, p. 25)². Esta línea de pensamiento, trata de hacer énfasis en dos aspectos.

El primer aspecto es que el trabajo doméstico femenino (que tiene una estrecha relación con la reproducción de la fuerza de trabajo), es una forma de producir valores de uso (cuidados, compras, limpieza, etc.) necesarios para el funcionamiento de la lógica del capital. Este es un fenómeno que no es privativo de este modo de producción en específico, pero que sí asume determinaciones históricas específicas dentro del modo de producción capitalista. Lo anterior, tiene su fundamento en que estos valores de uso sirven para producir y reproducir fuerza de trabajo en lo cotidiano y en lo generacional. Tanto el trabajo doméstico sexuado, como las interrelaciones que se establecen con la reproducción de la fuerza de trabajo como encargo exclusivo femenino, en el espacio de la familia patriarcal trabajadora, son analizados como elementos necesarios, funcionales al desarrollo del capitalismo como sistema. No perder de vista que sobre dicha problemática descansan relaciones de poder, de producción, de clases, pero también relaciones de género.

El segundo aspecto es comprender la relación género-capital, como parte de las relaciones entre la esfera doméstica y la esfera extradoméstica. En este sentido, es relevante destacar que la inserción de la mujer a la esfera de la producción social dentro del modo de producción capitalista (específicamente a partir de la Revolución Industrial) constituyó un paso de avance indiscutible con modos anteriores, pues la convirtió en fuerza de trabajo y con ello la posibilidad germinal de llevar a cabo su proceso de emancipación.

Sin embargo, ese proceso de liberación de la mujer y de alcanzar la igualdad no fraguaría debido a que la inserción de la mujer en el mundo de fuerza de trabajo se realizaría sobre la base de trabajos menos remunerados, precarios y a través de una fuerte incorporación al ejército industrial de reserva. Adicionalmente, esta inserción no significó una reducción de las responsabilidades y exigencia en la esfera privada; en la práctica se produjo una sumatoria o agregación entre trabajo doméstico y trabajo fuera de casa, con implicaciones negativas, debido a que las mujeres no sólo se vieron obligadas a realizar varias jornadas laborales al mismo tiempo; sino que además, con la duplicación de su jornada de trabajo se produce también una duplicación de su alienación como trabajadora, de la enajenación de su trabajo (tanto como mercancía-mujer, como mercancía-fuerza de trabajo); y por ende, una explotación redoblada.

La mujer trabajadora, en general, realiza su actividad de trabajo duplamente, dentro y fuera de casa. Y, al hacerlo, además de la duplicidad del acto de trabajo, ella es doblemente explotada por el capital. En espacio privado del hogar, ella invierte una gran cantidad de horas en el trabajo doméstico, con lo que contribuye a la reproducción, de esta esfera no directamente mercantil, en que se crean las condiciones indispensables para la reproducción de fuerza de trabajo de sus maridos, hijos(as) y de sí misma (y por ende también del capital). Sin esta esfera las condiciones de reproducción del sistema de metabolismo social del capital estarían bastante comprometidas, sino inviabilizadas.

Por su parte, Marx y Engels en su escrito de 1845 de La Ideología Alemana va a resaltar que la forma social de la vida productiva, tiene efectos determinantes en la estructura familiar y su funcionamiento. En este mismo trabajo, realiza un análisis de la división sexual del trabajo ${ }^{3}$ no como un resultado natural, sino como una construcción socio-histórica ${ }^{4}$. De esta forma,

Marx descubre que la división social y doméstica del trabajo es históricamente específica y, además, que el que realiza trabajo doméstico es siempre dominado por quien tiene su rol fuera del hogar, y que por tanto la división entre trabajo asalariado y trabajo doméstico promueve un patrón de dominación en las relaciones de género. (Haug, 2006, p. 5).

Por último, otros aspecto relevante es comprender la cuestión de que la relación entre producciónreproducción, y el trabajo económico-extraeconómico como un mecanismo o válvula de ajuste, en función de las necesidades del sistema en cada época histórica. En este sentido, Haug (2006, p. 331) destaca que "[...] el entrelazamiento de la explotación capitalista y una específica división del trabajo en relaciones de género históricas muestra que, entre otros tipos de opresión, la producción capitalista se basa en la opresión de la mujer." 
En este sentido Toledo (2017, p. 55,) plantea que: “[...] el Capitalismo usó la división sexual y la desigualdad de género, para estimular la competencia entre los trabajadores y bajar el nivel de salarios. La mujer es sinónimo de trabajo barato." Por su parte, otras autoras defienden la idea de que esta "[...] articulación entre el trabajo doméstico, como fuente de reproducción e procreación de fuerza de trabajo, y la producción social muestran como el uso de la fuerza de trabajo femenino es uno de los recursos para el capitalista expandir la extracción de plusvalía absoluta". (Toledo, 2017, p. 77).

De manera general, estos aspectos revelan el rol que juegan las relaciones de género para el proceso de acumulación capitalista. En otras palabras, se produce una articulación producción-reproducción y géneroclase en el capitalismo, que tienen como trasfondo tanto la explotación de clase, como la opresión de género. Igualmente destacar que en la medida en que se asumen las relaciones de género como relaciones sociales de producción, es posible entender las desigualdades de género como parte de las expresiones a nivel más concreto de la contradicción esencial capital-trabajo; y por ende se comprende también que su superación no puede ser lograda esencialmente en los marcos del sistema capitalista. De esta perspectiva, la hegemonía tanto del patriarcado y como de las relaciones capitalistas de producción, se convierten en fenómenos complementarios, que se fortalecen mutuamente.

\section{Capitalismo globalizado: la internacionalización de las desigualdades y opresiones de género en la contemporaneidad}

La globalización de la economía mundial debe ser entendida esencialmente como la forma contemporánea de internacionalización de las relaciones capitalistas de producción. Su contextualización histórica se encuentra asociada a la necesidad del reacomodo del patrón de acumulación, que acontece a finales de los años sesenta y principio de la década del setenta, debido a la crisis estructural ${ }^{5}$ del sistema capitalista a partir de estos años.

Es un proceso que implica cambios sustanciales en casi todas las facetas de la vida social. No obstante, se distinguen tres esferas que se constituyen en elementos centrales de esta transformación y que cualifican distintivamente a este proceso: la globalización productiva, la globalización comercial y la globalización financiera ${ }^{6}$. Los cambios en estas tres esferas se encuentran interconectados, y en su conjunto tributan al ajuste de patrón de acumulación del capital en este nuevo escenario. El carácter sistémico y de totalidad de las transformaciones fue innegable; aun así, sin obviamente tocar los elementos estructurales y esenciales que daban lugar a dichas contradicciones.

A su vez, es importante destacar que la globalización de la economía mundial no es sólo un proceso, sino también un proyecto de la lógica del capital, que tiene un trasfondo político e ideológico. El neoliberalismo es el proyecto ideológico que sustenta el proceso de globalización. La compleja fusión de ambos aspectos es lo que en este artículo denominaremos en lo adelante como globalización neoliberal. Es por ello, que se defiende la idea de que la globalización neoliberal de la economía mundial debe ser analizada simultáneamente como un proceso de ajuste, tanto del portafolio de valorización, como de la ideología que sustenta al proyecto societario capitalista. Ambos ajustes, el de la acumulación y el de la ideología, son una unidad irreductible.

El reajuste del mecanismo económico capitalista acontecido a partir de la globalización neoliberal, puede ser sintetizado a partir de tres elementos básicos: la liberalización y desregulación (como nueva forma de regulación) de: mercados, capitales, fronteras; la privatización, sobre la idea de potenciar un sector privado que es eficiente y estable por si solo (según el discurso neoliberal); y la aparente minimización de papel los EstadosNación, en pos de un nuevo orden mundial, cada vez más pautado por instituciones y poderes supranacionales.

Dentro de este proceso, el llamado mercado de trabajo ${ }^{7}$ fue tal vez el menos globalizado de todos, pero no por ello el menos impactado; debido al fuerte proceso de flexibilización a que ha sido sometido. Lo anterior se concreta en una serie de transformaciones asociadas a este proceso como: la potenciación de obreros polivalentes, el desarrollo del trabajo a distancia a través de internet, la eliminación de algunas barreras a la movilidad social y laboral, la subcontratación, los contratos a tiempo parcial o temporales, entre otros cambios.

Adicionalmente, los reajustes y la desregulación aplicados en el mercado de fuerza de trabajo, han eliminado importantes regulaciones protectoras e instituciones con la excusa de que constituyen barreras para la flexibilidad y la competitividad de las economías. De esta manera, las exigencias de la nueva dinámica económica global (financiera, desregulada, rentista), ha reconfigurado los mercados de fuerza de trabajo a través de la flexibilización, la segmentación, y la dualización. Lo cual a su vez ha conllevado a que aquellas personas que se logran insertar en la esfera laboral, trabajen en condiciones de mayor incertidumbre (con todos los impactos emocionales-psicológicos que ellos conlleva) en cuanto a la conservación de su empleo; y por ende, más expuestos a las distintas formas de opresión y explotación impuestas por el capital.

Es por ello que, se plantea que la globalización neoliberal ha multiplicado la lógica del capital más allá del mercado de fuerza trabajo, pero partiendo de él, en otras palabras, ha potenciado la subordinación estructural 
del trabajo al capital con todas sus implicaciones fuera del mundo del trabajo; abarcando casi todas las esferas de la vida y del mundo. Situación que ha convertido a la creciente exclusión, precarización, flexibilización, enajenación y explotación, en características no ya exclusivas de los que se encuentran empleados; sino comunes a casi toda la sociedad.

Por su parte, dentro del análisis de género en el mundo del trabajo en el contexto de la globalización, las repercusiones para las mujeres han sido diferenciadas, por su relevancia para esta investigación, a continuación se profundiza en esta perspectiva de análisis.

En este sentido, se defiende la idea de que con la globalización ocurre una agudización de la subordinación/ opresión impuesta socialmente a las mujeres, debido a la mayor funcionalidad, en relación a la lógica del capital, que adquieren a partir de las formas de exclusión/inserción laboral que mayormente va a distinguir a las mujeres en esta etapa. Según Girón (2009, p. 80):

La era post Bretton Woods, cuya caracterización más visible han sido las crisis económicas y financieras, los planes de estabilización y las reformas económicas. Precisamente, en este proceso, son las mujeres el eje del cambio en las relaciones de producción. Las mujeres son en dicha rearticulación de la estructura productiva global quienes avanzan en el mercado como fuerza de trabajo. Las mujeres pasan a ser proveedoras de los ingresos familiares ocupándose hasta en dos y tres jornadas laborales. Muchas veces, además de su jornada laboral en la casa, realizan actividades en el mercado informal. Pasan a ser proveedoras de sus familias y también jefas de hogar. La sociedad castiga a sus mujeres marginándolas del acceso al crédito, el trabajo formal, el derecho a una pensión digna, los servicios de salud, vivienda y por supuesto educación.

El impacto de las políticas financieras, fiscales y monetarias transformó las estructuras de producción y al mercado de fuerza de trabajo, potenciando una mayor participación de la fuerza laboral femenina; pero sin que se produjeran cambios sustanciales en pos de la igualdad de género.

Para Ricardo Antunes ${ }^{8}$, la restructuración productiva conllevó a una subproletarización intensificada, presente en la expansión del trabajo parcial, temporario, precario, subcontratado, tercierizado. Siempre en el marco de una reducción de puestos de trabajo para ambos, hombres y mujeres, ha habido una mayor demanda de mano de obra femenina ara ocupar precisamente este tipo de trabajo. (Toledo, 2017, p. 58).

Ejemplo lo anteriormente expuesto, es que crece la inserción laboral; sin embargo, la presencia de las mujeres en sectores tradicionalmente masculinos, y especialmente en posiciones de mayor responsabilidad y de toma de decisiones a alto nivel (y por ende de poder) históricamente asignadas a los hombres, no se incrementa al mismo ritmo. En definitiva, en casi todas las regiones del mundo aumentó la participación laboral de las mujeres; no obstante, también se constata que las condiciones bajo las cuales se insertan las féminas en el mercado de fuerza de trabajo (segregación horizontal y vertical, discriminación salarial, informalidad, condiciones de seguridad y proyección en el empleo, etc.) no solo continúan siendo desfavorables, sino que con la globalización se han deteriorado aún más.

La tendencia anterior que refleja la expansión del empleo femenino, se compensa negativamente por otro lado con el hecho de que el ajuste estructural y las políticas neoliberales que acompañaron a la globalización fueron particularmente dañinas para las mujeres. Estos llevaron a recortes en los empleos del sector público, donde trabajaban en mayoritariamente mujeres, y también a recortes en las políticas/programas sociales que beneficiaban a las mismas y les permitían, en cierto sentido, organizarse para trabajar. Las privatizaciones, la desregulación de los mercados y la reestructuración económica contribuyeron a establecer condiciones para la expansión de las actividades informales y condiciones precarias de trabajo en muchos países. Según Kudva y Benería (2007, pp. 87-154),

[...] un tema dominante y recurrente en la literatura sobre el ajuste estructural y el género es el creciente feminización de los mercados [...] que ocurre bajo ajuste estructural a medida que el empleo se vuelve más flexible, eventual e informal. Se dice que un ajuste estructural ha llevado a una intensificación del trabajo reproductivo de las mujeres, el aumento de la participación femenina y el deterioro de las condiciones de trabajo. [...] Ya sea que se llame reestructuración y liberalización, en la primer mundo, o ajuste estructural y la gestión de la deuda en las políticas neoliberales del tercer mundo, ambas se han caracterizado por retirar el apoyo público para los servicios básicos y sociales, ha intensificado la explotación del trabajo de las mujeres. Son políticas que asumen la disponibilidad infinita y el trabajo elástico de las mujeres; se basan en el trabajo gratuito y mal pagado de las mujeres en el hogar y en la comunidad para compensar la erosión de la esfera pública y el abandono de sus responsabilidades. 
De esta forma, aunque crece la inserción laboral para las mujeres, lo hace al mismo también la desocupación femenina, pues el tipo de ofertas de trabajo potenciadas con la globalización están caracterizadas por su concentración en empleo de menor calidad y con una fuerte presencia en la economía informal. Las empresas multinacionales demandan para la producción off shore, trabajo barato y flexible. Las mujeres permanecen excluidas en su gran mayoría de los trabajos más estables y mejor remunerados.

Lo que aparentemente se presenta, como dos procesos contradictorios - mayor inserción laboral y al mismo tiempo, mayor desigualdad y exclusión de las mujeres en el ámbito laboral - puede ser explicado a través del binomio: feminización del trabajo (incremento de las tasas de participación laboral femenina) vs. feminización del reajuste laboral (asociado al marcado efecto negativo sobre las mujeres del proyecto y proceso de la globalización neoliberal). Esencialmente, estos procesos no son en sí mismos contradictorios, sino que responden a la necesidad de la lógica de capital de restructurar tanto el empleo como el mercado de fuerza de trabajo en tiempos de la globalización neoliberal, lo cual tiene un efecto para toda la sociedad, pero relativamente más marcado (negativamente) para las mujeres.

Este proceso de reajuste con rostro de mujer del mundo del trabajo, se concreta en expresiones como la feminización de: el trabajo informal; los trabajos no remunerados asociados al hogar; la tercerización; los trabajos a tiempo parcial o de manera temporal; la mayor precarización del empleo, los menores salarios; la subcontratación; el desempleo; la desigualdad salarial; la segregación del mercado laboral; la desocupación; la violencia en el ámbito laboral; la pobreza, entre otras contradicciones sociales.

Lo novedoso de esta etapa de la globalización neoliberal no son sólo las formas de manifestación concreta de cada uno de estos fenómenos, sino también los entrecruzamientos entre ellos se generan y su elevada funcionalidad a las nuevas exigencias de modelo de acumulación capitalista global. En otras palabras, no son procesos aislados, inconexos; sino que su articulación responde precisamente a las necesidades de acumulación del sistema. De esta forma, la creciente desigualdad de las mujeres en el mercado laboral asegura una "[...] mano de obra barata para un conjunto de actividades y formas de relación laboral necesarias para el funcionamiento de la economía global." (Todaro, 2000).

En este sentido, Dantas y Cisne (2017, p. 86) analizan que,

[...] la ideología de que las mujeres, al conquistar el mercado de trabajo, alcanzaron la emancipación. Entendemos que, aunque hayamos avanzado, inclusive ocupando determinados puestos de trabajo históricamente masculinos, todavía no superamos la lógica jerárquica de la división sexual del trabajo que estructura la explotación del trabajo en esta sociedad. Además de la desigualdad salarial, los mayores índices de desempleo e de precarización en el trabajo afectan más fuertemente a las mujeres. Resaltamos, todavía, la apropiación por parte del capital, de habilidades socialmente construidas en las mujeres para la intensificación de la explotación, como, por ejemplo, la polivalencia y la mejor adecuación al trabajo realizado a domicilio, expresiones de transformaciones contemporáneas en el mundo del trabajo.

Adicionalmente, una buena parte de los éxitos, en cuanto al aumento de productividad, de estos programas de ajuste neoliberal, no muestran las transferencias de costos desde el mercado a los hogares; y específicamente a las mujeres. Al respecto, Vargas (2009, p. 10) señala que:

En todos los procesos de ajuste estructural, las mujeres han funcionado como un factor oculto de equilibrio para absorber los shocks de los programas de ajuste de la economía, tanto intensificando el trabajo doméstico para compensar la disminución de los servicios sociales por la caída del gasto público, como por el hecho de que la privatización de los sistemas de seguridad social ha incidido en mayor medida en las mujeres por su papel en la reproducción (costos sociales de la maternidad asumidos individualmente, por ejemplo). Así, su posición en la familia y en el mercado de trabajo las ubica como parte de la estrategia desreguladora del mercado.

Una de las formas en que se expresa dicho proceso es que se innova en relación a la conciliación (de manera forma) entre los espacios públicos y privados para las mujeres, "[...] participan en mayor grado como proveedoras de ingreso familiar en la economía informal o en la economía formal; pero sin desprenderse de su trabajo doméstico." (Girón, 2009, p. 86).

En este sentido, Todaro (2000) afirma que las responsabilidades familiares pueden contribuir a volver a las mujeres más vulnerables a la precarización de los empleos, ya que muchas veces deben aceptar trabajos de peor calidad, con menor protección laboral y de seguridad social, a cambio de flexibilidad para compatibilizar trabajo doméstico y trabajo remunerado. Esto permite transformar esta vulnerabilidad de las mujeres en parte de la estrategia de acumulación del sistema. 
Finalmente, se destacan algunos datos que ilustran el complejo escenario que enfrentan las mujeres en la región de América Latina y el Caribe, a casi tres décadas de aplicación de las contrarreformas neoliberales. De esta manera, se han tratado de seleccionar estadísticas actuales que ejemplifiquen los fenómenos analizados realizados a lo largo de este artículo. En todos los casos la fuente de información son publicaciones diversas de la Comisión Económica para América Latina y el Caribe (CEPAL).

En este sentido, la CEPAL sistemáticamente en los últimos años ha venido destacando problemas urgentes en relación a la igualdad de género que presenta la región. Entre los aspectos críticos apuntados resaltan: menores tasas de participación laboral y mayores tasas de desocupación; la falta de ingresos propios y suficientes; desigualdad salarial en detrimento de las féminas, segregación horizontal y vertical; existencia de un techo de cristal para las mujeres; la sobrecarga de trabajo doméstico y de cuidado no remunerado; la sobrerrepresentación en los hogares pobres; la violencia contra las mujeres; las dificultades para decidir libremente sobre su vida sexual y reproductiva; la insuficiente representación en los espacios de poder; entre otros. A continuación, se presentan algunas estadísticas:

En pleno siglo XXI, la pobreza en el continente latinoamericano aún tiene rostro de mujer. "Si en 2002 por cada 100 hombres viviendo en hogares pobres había 107 mujeres, en 2014 esta cifra aumenta a 118 mujeres. Paradójicamente, en un período donde la pobreza en la región cayó del 43,9\% al 28,2\%." (CEPAL, 2016a). "Además, un tercio de las mujeres latinoamericanas (29\%) no logra generar ingresos y son económicamente dependientes y cerca de la mitad no tiene vínculo con el mercado laboral." (CEPAL, 2017b);

El "[...] 29\% de las mujeres no tiene ingresos propios (12,5\% de los hombres) y $26 \%$ percibe un salario inferior al mínimo (18,3\% de los hombres). En el otro extremo, solo un $7 \%$ de las mujeres latinoamericanas recibe ingresos iguales o superiores a cuatro salarios mínimos, porcentaje que se eleva a $16 \%$ en el caso de los hombres. [...] A pesar de que se redujo la brecha salarial en 12,1 puntos porcentuales entre 1990 y 2014, las mujeres reciben en promedio solo el $83,9 \%$ de lo que reciben los hombres. La mayor diferencia se presenta en la población de mayor nivel educativo [...]" (CEPAL, 2016a);

De manera general, "las mujeres reciben en promedio solo 83,9 unidades monetarias por cada 100 unidades monetarias percibidas por los hombres [...] Si se comparan las remuneraciones recibidas por ambos sexos según años de estudio, se observa que ellas pueden ganar hasta 25,6 por ciento menos que sus pares masculinos en similares condiciones [...]" (CEPAL, 2016b);

"En la región, 78,1\% de las mujeres que están ocupadas se desempeñan en los tres sectores definidos como de baja productividad (agricultura, comercio y servicios sociales, comunales y personales), lo que implica peores remuneraciones, menor cobertura de la seguridad social y menor contacto con las tecnologías y la innovación." (CEPAL, 2016a);

"Si bien durante la última década los indicadores del mercado de trabajo presentan mostrado una evolución positiva, la tasa de participación laboral femenina se ha estancado en torno al 53\% y el $78,1 \%$ de las mujeres que están ocupadas lo hacen en sectores definidos por la CEPAL como de baja productividad, lo que implica peores remuneraciones, baja cobertura de la seguridad social y menor contacto con las tecnologías y la innovación." (CEPAL, 2017c);

"[...] las tasas de desempleo de las mujeres son sistemáticamente mayores que las de los hombres [...]. Entre 2002 y 2013, la tasa de desempleo en América Latina acumuló un descenso de 2,8 puntos porcentuales, pero a partir de 2015 esta tendencia se ha revertido. [...] en 2015 la tasa de desempleo alcanzó 7,4\%, siendo las mujeres las más perjudicadas: el 8,6\% de ellas estaba desempleada en comparación con el 6,6\% de los hombres." (CEPAL, 2017c);

"[...] cuando se analiza la evolución de la tasa de desocupación por país es el mantenimiento de la brecha de desocupación entre los hombres y las mujeres en 2017 [...]. Si bien durante la fase contractiva de 2016 las tasas de desocupación masculinas tendieron a aumentar, en promedio, menos que las femeninas [...]" (CEPAL \& Organización Internacional del Trabajo [OIT], 2018, p. 11);

"[...] las mujeres realizan entre el $71 \%$ y el $86 \%$ del trabajo no remunerado total que demandan los hogares, dependiendo del país. Más aún, la mitad de las mujeres en cuyo hogar hay niños o niñas menores de 7 años se encuentra fuera del mercado laboral." (CEPAL, 2016a);

"La inserción laboral en la edad activa se caracteriza por grandes brechas entre hombres y mujeres, a causa de la distribución desigual de las tareas domésticas e interrupciones en la trayectoria de trabajo en un mundo productivo y laboral cada vez más volátil.” (CEPAL \& OIT, 2018, p. 19). “[...] para el conjunto de los ocupados, la proporción de mujeres es menor que la de hombres con respecto a los empleadores, los trabajadores por cuenta propia y los asalariados y mayor en el empleo doméstico y el trabajo familiar no remunerado." (CEPAL \& OIT, 2018, p. 35); 
"[...] la presencia de mujeres no supera el $30 \%$ en los espacios de toma de decisiones en los poderes del Estado: órganos legislativos (29,9\%), cortes supremas (29,1\%), concejalías (27,3\%), gabinetes ministeriales $(23,9 \%)$ y alcaldías $(12,3 \%)$." (CEPAL, 2016a). "[...] la presencia de mujeres en los ámbitos de poder todavía no supera el 30\%." (CEPAL, 2017a).

\section{Reflexiones finales}

De manera general, se evidencia que la cuestión de género es un tema de gran relevancia en la actualidad. El capitalismo en su actual contexto de globalización neoliberal ha exacerbado, fetichizado y profundizado aún más las formas de opresión de género y de explotación de clase; por lo cual ante una complejización cada vez mayor de este fenómeno, es también necesaria una mirada cada vez más integradora y crítica desde el marxismo.

La temática de género es una problemática no resuelta por el llamado desarrollo capitalista, ni por las experiencias de transición al socialismo, al menos en toda su expresión. Constituye pues un aspecto central para entender, y al mismo tiempo cuestionarse, las formas concretas que hoy en día asumen aspectos como: el patriarcado, la división sexual y social del trabajo, los procesos de producciónreproducción social, la ideología, la política, la ley, la religión, la moral, la sexualidad, la dominación/ opresión, las clases sociales, la explotación, etc.

El análisis marxista de las cuestiones de género, a partir de una crítica desde sus fundamentos, permite - en la medida que las relaciones de género son también entendidas como manifestaciones de las relaciones sociales de producción - poner al descubierto su vinculación con los procesos de acumulación capitalista y su funcionalidad a la lógica del capital. Adicionalmente, en la medida en que esta idea se comparte, también se avanza en la compresión de que no basta con transformar las formas y las instituciones en que se concreta la opresión, la desigualdad y la discriminación de género; es preciso ir a las determinaciones de última instancia, a la esencia, y mudar el metabolismo social del capital; y por ende, las premisas que las sustentan. Solo un proyecto (socialista) que se proponga y logre transformar por oposición, ruptura y superación la lógica del capital, sentará las bases para una igualdad sustantiva y una verdadera emancipación de los seres humanos.

\section{Referencias}

Antunes, R. (1995). Adeus ao Trabalho? São Paulo: Cortez.

Anzorena, C. (2008). Estado y división sexual del trabajo: las relaciones de género en las nuevas condiciones del mercado. Utopía y Praxis Latinoamericana, 13(41), abr./jun.

Benería, L. (1984). Reproducción, producción y división sexual del trabajo. Santo Domingo: CIPAF.

Benería, L. (1995). Towards a greater integration of gender in economics. World Development, 23(11), 1839-1850.

Benería, L. (2005). Género, desarrollo y globalización: Por una ciencia económica para todas las personas. Barcelona: Hacer.

Comisión Económica para América Latina y el Caribe. (2016a, 24 de octubre). CEPAL llama a aplicar medidas innovadoras y efectivas para garantizar los derechos de las mujeres en América Latina y el Caribe. Recuperado de https://www.cepal.org/es/comunicados/cepalllama-aplicar-medidas-innovadoras-efectivas-garantizar-derechos-mujeres-america

Comisión Económica para América Latina y el Caribe. (2016b, 8 de marzo). Pese a avances en el nivel educacional de las mujeres, persiste brecha salarial de género en la región. Recuperado de https://www.cepal.org/es/comunicados/pese-avances-nivel-educacional-mujerespersiste-brecha-salarial-genero-la-region

Comisión Económica para América Latina y el Caribe. (2017a, 20 de junio). Alicia Bárcena llama a las mujeres a romper el techo de cristal a través de la emancipación y la autonomía. Recuperado de https://www.cepal.org/es/comunicados/alicia-barcena-llama-mujeres-rompertecho-cristal-traves-la-emancipacion-la-autonomia

Comisión Económica para América Latina y el Caribe. (2017b, 5 de octubre). Celebran en La Habana 40 años de avances en igualdad y derechos de las mujeres de la región con foco en los retos pendiente. Recuperado de https://www.cepal.org/es/comunicados/celebran-lahabana-40-anos-avances-igualdad-derechos-mujeres-la-region-foco-retos

Comisión Económica para América Latina y el Caribe. (2017c, 7 de marzo). CEPAL: Tasa de participación laboral femenina se ha estancado en torno al 53\% en la región y persisten sesgos de género en el mercado de trabajo. Recuperado de https://www.cepal.org/es/ comunicados/cepal-tasa-participacion-laboral-femenina-se-ha-estancado-torno-al-53-la-region

Comisión Económica para América Latina y el Caribe, \& Organización Internacional del Trabajo. (2018). Coyuntura Laboral en América Latina y el Caribe, (18). Recuperado de https://repositorio.cepal.org/bitstream/handle/11362/43603/1/S1800398_es.pdf

Dantas, M. F. \& Cisne, M. (2017). Trabalhadora não é o feminino de trabalhador: superexploração sobre o trabalho das mulheres. Argumentum, 9(1), 75-88, enero/abr.

Gimenez, M. (2005). Capitalism and the oppression of women: Marx revisited. Science \& Society, 69(1), 11-32. Recuperado de http:/ 
/citeseerx.ist.psu.edu/viewdoc/download?doi=10.1.1.128.3046\&rep=rep1\&type=pdf

Girón, A. (2009). Género, globalización y desarrollo. In A. Girón (Coord.), Género y globalización (pp. 77-97). Buenos Aires: CLACSO. Recuperado de http://biblioteca.clacso.edu.ar/ar/libros/grupos/giron/GyG.pdf

Haug, F. (2006). Hacia una teoría de las relaciones de género. In A. A. Boron, J. Amadeo, \& S. González (Orgs.), La teoría marxista hoy: Problemas y perspectivas (pp. 327-340). Buenos Aires: CLACSO. Recuperado de http://resistir.info/livros/ boron_la_teoria_marxista_hoy.pdf

Kudva, N., \& Benería, L. (2007). Rethinking informalization: poverty, precarious jobs and social protection. New York: Cornell University Press. Recuperado de https://ecommons.cornell.edu/bitstream/handle/1813/3716/Rethinking\%2 OInformalization.pdf;sequence=1

Mandel, E. (1982). O capitalismo tardio. São Paulo: Abril Cultural.

Mészáros, I. (2011). A crise estrutural do capital (2a ed.). São Paulo: Boitempo.

Molyneux, M. (1994). Más allá del debate sobre el trabajo doméstico. In C. Borderías, C. Carrasco, \& C. Alemany (Orgs.), Las mujeres y el trabajo: rupturas conceptuales (pp. 111-150). Barcelona: Icaria.

Navarro, V. (1997). Neoliberalismo y estado de bienestar. Barcelona: Ariel.

Pérez Soto, O. (2009). Internacionalización del capital: respuesta socialista. La Habana: Félix Varela.

Todaro, R. (2000). Aspectos de género de la globalización y la pobreza. Recuperado de http://www.un.org/womenwatch/daw/csw/ todaro.htm

Todaro, R. (2004). Ampliar la mirada: trabajo y reproducción social. In R. Todaro, \& S. Yañez (Eds.), El trabajo se transforma: Relaciones de producción y relaciones de género (pp. 15-32). Santiago: Centro de Estudios de la Mujer. Recuperado de http:// bdigital.unal.edu.co/47315/1/9567023131.pdf

Toledo, C. (2017). Gênero e classe. São Paulo: Sundermann.

Vargas, V. (2009). Prólogo. In A. Girón (Coord.), Género y globalización (pp. 9-12). Buenos Aires: CLACSO. Recuperado de http:// biblioteca.clacso.edu.ar/ar/libros/grupos/giron/GyG.pdf

Yañez, S. (2004). La flexibilidad laboral como nuevo eje de la producción y la reproducción. In R. Todaro, \& S. Yañez (Eds.), El trabajo se transforma: Relaciones de producción y relaciones de género (pp. 35-73) Santiago: Centro de Estudios de la Mujer. Recuperado de http://bdigital.unal.edu.co/47315/1/9567023131.pdf

\section{Notas}

1 La distinción entre producción y reproducción es una diferenciación esencialmente metodológica al interior de un único proceso que constituye la (re)producción de la vida social. En este sentido, Todaro (2004, p. 20) plantea que: "La reproducción social es el proceso dinámico de cambio vinculado a la perpetuación de los sistemas sociales, e involucra tanto factores económicos como ideológicos, políticos y sociales en un pro-ceso de mutua influencia. Se pueden distinguir tres aspectos de la reproducción: la reproducción social, la reproducción de la fuerza de trabajo y la reproducción biológica, que implican diferentes niveles de abstracción teórica." De esta forma, el concepto de reproducción se asumen como un proceso dinámico relacionado con la perpetuación de los sistemas sociales en el cual el papel de las mujeres es decisivo. La producción, el trabajo y la división de este último por sexos adquieren una dimensión diferente desde esta perspectiva: por una parte, deben analizarse en sus interrelaciones y, por otra, no son estáticos, se integran en un sistema socio-económico en movimiento, en continua transformación.

2 Con ello no se pretende trasmitir la idea de que la división sexual del trabajo sea un fenómeno novedoso del Capitalismo, por el contrario es un proceso que tiene su génesis en los propios albores de la humanidad; no obstante, se pretende hacer énfasis en que el modo de producción capitalista ha utilizado de forma funcional dicha división sexual del trabajo para dar respuesta a variadas exigencias del proceso de valorización del capital en diferentes momentos históricos, y que este fenómeno ha asumido bajo el Capitalismo determinaciones histórico-concretas particulares.

3 Según Molyneux (1994) y Benería (1984, 1995, 2005), la división sexual del trabajo se refiere a los determinados roles, espacios, actividades asignadas y que se esperen realicen las mujeres y hombres de las edades diferenciadamente. De este modo, se construye una división jerárquicay binaria del trabajo, separando reproducción de producción.

4 Idea que Engels también desarrolla en la obra El origen de la familia, la propiedad privada y el Estado, publicada en 1844. Esta obra constituye una de las primeras concepciones materialistas de la opresión de las mujeres. En ella Engels trasciende el análisis desde la biología de la opresión de la mujer y lo explica a través de aspectos históricos y culturales; con lo cual implícitamente se llega a la conclusión de que si la opresión no es algo natural, puede ser superada.

5 Según Mandel (1982), Mészáros (2011) y otros marxistas, la crisis estructural del capital, que comienza en este período no tiene solución (efectiva/ esencial) hasta nuestros días; $y$ no puede hacerlo porque implica superarla implicaría una solución estructural fuera de los marcos del metabolismo social del capital.

6 “[...] es importante que clarifiquemos el concepto de globalización puesto que con excesiva frecuencia se están mezclando diferentes conceptos, presentándolos bajo una misma rúbrica. De ahí que para fines de esta exposición se quisiera desagregar este concepto en sus diferentes componentes, diferenciando la globalización del comercio, de la globalización del capital productivo y estás a su vez de la globalización del capital financiero." (Navarro, 1997, p. 205). Un enfoque similar puede ser consultado en Pérez Soto (2009). 
7 Se coloca en cursiva como una forma de crítica al uso de esta expresión (con todo su trasfondo epistemológico y axiológico) por parte de la economía convencional. Desde la perspectiva marxista lo que constituye una mercancía es la fuerza de trabajo, y no el trabajo que es un resultado/ materialización de la capacidad de trabajo de la fuerza productiva; por ello desde crítica marxista, en todo caso se debería hablar de mercado de fuerza de trabajo.

8 Consultar Antunes (1995).

9 Esta institución perteneciente a las Naciones Unidas, tiene un discurso esencialmente reformista y conciliador en términos de análisis y propuestas. No obstante - pese a no cuestionarse la posibilidad del desarrollo para los países dependientes de América Latina, ni colocar en entredicho la posibilidad de una transformación estructural y sustantiva en los marcos de la lógica del capital-sistemáticamente publica y analiza temas socioeconómicos de la región, entre ellos una amplia variedad de publicaciones sobre género. La crítica que realiza (desde lo descriptivoaparencial) permite identificar algunas tendencias y contradicciones interesantes en este debate.

\section{Arelys Esquenazi Borrego}

arelyseb@gmail.com

Máster en Economía con mención en Política Económica por la Facultad de Economía de la Universidad de La Habana (UH)

Doctoranda del Programa de Post-Graduación en Política Social de la Universidade Federal do Espírito Santo (UFES)

\section{UFES}

Av. Fernando Ferrari, 514 - Goiabeiras

Vitória - Espírito Santo - Brasil

CEP: 29.075-073

\section{Agencia financiadora}

No se aplica.

Contribuciones de los autores

No se aplica.

\section{Consentimiento para publicación \\ No se aplica.}

\section{Conflicto de intereses}

No existe conflicto de intereses. 